\title{
Complementary split-ring resonator-based deflecting structure
}

\author{
I. McGregor ${ }^{1,2}$ and K. M. Hock ${ }^{1,2}$ \\ ${ }^{1}$ Department of Physics, University of Liverpool, Liverpool L69 7ZE, United Kingdom \\ ${ }^{2}$ Cockcroft Institute, Daresbury Laboratory, Warrington WA4 4AD, United Kingdom
}

(Received 29 June 2013; published 16 September 2013)

\begin{abstract}
We describe full wave simulation results for a compact, novel deflecting structure operating around $2 \mathrm{GHz}$. The structure consists of a pair of elliptical complementary split-ring resonators (CSRRs) etched into a copper rectangular waveguide along with outer rectangular cavities to maintain the vacuum. Our design utilizes the strong electric fields created by the resonators to impart a transverse deflection to particle bunches traveling through the structure. Simulations suggest a total deflecting voltage of $0.132 \mathrm{MV}$ and a transverse accelerating gradient of $1.55 \mathrm{MV} / \mathrm{m}$ for only $4.42 \mathrm{~kW}$ of applied rf power. We also present, as a design aid, a simplified, approximate analysis of the operating frequency.
\end{abstract}

DOI: 10.1103/PhysRevSTAB.16.090101

PACS numbers: 29.20.-c, 81.05.Xj

\section{INTRODUCTION}

Traditionally, the trajectories of relativistic particle beams are deflected by strong magnetostatic fields. However, when the particle beams are propagating at speeds much below the speed of light, the Lorentz force due to practical magnetic fields becomes so weak that it may be advantageous to use electric fields to impart deflections. Deflectors which utilize electric fields come in two varieties: electrostatic and alternating current. The electrostatic types typically find application in E-beam lithography where the beam is continuous during operation. For example, in [1] a comparison of the performance of six types of electrostatic deflector is given; each instance differing in geometry and number of poles. The alternating field-type deflectors are better suited to particle accelerator applications as greater field strengths can be obtained and they are compatible with the bunched nature of the beam. A common type of alternating field deflector is one similar to the ubiquitous multicell pillboxlike accelerating cavities but operated with TE modes instead of accelerating TM modes. For example, in [2] simulation results for a $\pi$ mode multicell cavity operating around $3 \mathrm{GHz}$ are presented. It obtains a deflecting voltage of 4.9 MV for an input power of $5 \mathrm{MW}$. This corresponds to a transverse accelerating gradient of $19.6 \mathrm{MV} / \mathrm{m}$ or, if expressed in the form gradient $(\mathrm{MV} / \mathrm{m})=k \sqrt{P_{\text {in }}}(\mathrm{MW})$ (where $P_{\text {in }}$ is the power in the applied excitation signal), to a $k$ value of 8.77 . Another example of a multiple-cell rf deflector, this time operating in the $2 \pi / 3$ mode and also operating around $3 \mathrm{GHz}$, is given in [3]. This cavity achieves a transverse accelerating gradient of $0.51 \mathrm{MV} / \mathrm{m}$ but at a much lower input power of $100 \mathrm{~kW}$ or with $k=1.6$. Both of

Published by the American Physical Society under the terms of the Creative Commons Attribution 3.0 License. Further distribution of this work must maintain attribution to the author(s) and the published article's title, journal citation, and DOI. the referenced deflecting cavities use normal-conducting copper-as is the case in the present work.

In this paper, we describe how it is possible to achieve useful deflecting voltages by using complementary splitring resonators (CSRRs) to magnify the electric fields in a rectangular waveguide. CSRRs are normally used as metamaterial elements to provide an effective negative permittivity [4]. They are "complementary" to the better known split-ring resonators [5] in the sense that, where in the SRR there is metal there is an absence of metal in the CSRR and vice versa. They are the circuit dual of SRRs in the sense that SRRs are characterized by resonant magnetic fields (and provide a negative effective permeability) and CSRRs by resonant electric fields (and yield a negative effective permittivity). The resonators are etched into a copper rectangular waveguide (one in the lower wall and one in the upper) propagating the dominant mode and are surmounted by rectangular cavities. The CSRRs in this work differ from those normally used in that they are elliptical rather than circular. This modification allows the deflecting field to be "stretched" in one plane and the total deflecting voltage made greater than that achieved by a circular CSRR geometry. By keeping the minor radius small, the resonance frequency can be kept higher than if a circular resonator with a large radius is used.

\section{DESCRIPTION OF THE DEFLECTING STRUCTURE}

We begin by describing the geometry of the elliptical CSRRs. Figure 1 shows a top view. It can be seen that the uniform portions of the structure resemble coplanar waveguide $(\mathrm{CPW})$ transmission lines with ground to ground spacing $w+2 s$ and a signal trace of width $w$. There are also two shunt inductive connections to the "ground planes" on the west and east side of the structure. The structure has a thickness $t$ (into the page). The resonator is further characterized by the radii $r_{x}$ and $r_{z}$. When excited by an E-field pointing into the page, and at a particular 


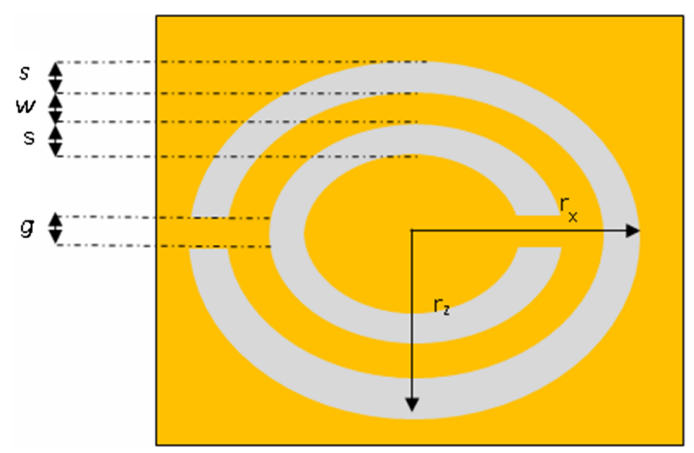

FIG. 1. Top view of the elliptical CSRR.

frequency dependent on the geometry of the CSRR, an electric monopole is created with a magnified electric field. It is this property that we utilize in our structure to create a strong E-field between two opposing CSRRs. This field can then be used to impart a transverse deflection to, say, a proton bunch traversing a path connecting the two inductive connections.

Figure 2 shows two views of the proposed deflecting structure. CSRRs etched into the upper and lower walls of a rectangular waveguide (of dimensions $a \times b$ ) propagating a $\mathrm{TE}_{10}$ mode are excited at their resonance frequency and produce a strong uniform field in the gap between. In general, this frequency may differ from the free-space resonance frequency owing to the resonators' proximity to each other and to the walls of the rectangular cavity. Two inductive irises are placed immediately before and after the CSRRs in the direction of rf power flow. Depending on the relative size of the waveguide width, $a$, and the CSRR major radius $r_{x}$, fields of opposite polarity to that desired to deflect the beam could be created if the irises are absent. The irises shield the particle bunches from such effects and
TABLE I. Geometrical parameters for designed structure (see Figs. 1 and 2) with operating frequency of $2.037 \mathrm{GHz}$.

\begin{tabular}{lccc}
\hline \hline Parameter & Value $(\mathrm{mm})$ & Parameter & Value $(\mathrm{mm})$ \\
\hline$r_{x}$ & 14 & $a$ & 85 \\
$r_{z}$ & 7 & $b$ & 8 \\
$w$ & 1.5 & $l_{\text {cav }}$ & 20 \\
$s$ & 1.5 & $h_{\text {cav }}$ & 9.5 \\
$g$ & 1 & $x_{\text {iris }}$ & 14 \\
$t$ & 1 & $z_{\text {iris }}$ & 22 \\
\hline \hline
\end{tabular}

ensure a unipolar field. These inductive irises, a distance $z_{\text {iris }}$ apart and with an iris width $x_{\text {iris }}$, when coupled to the CSRRs, impart a bandpass-like response to the structure. The maximum deflecting field occurs at the same frequency as maximum power transmission from input to output port.

Without the inductive irises, there would be a transmission null at the frequency at which maximum field strength occurs between the CSRRs. The integrated $y$ component of the electric field (deflecting voltage) would also be smaller in magnitude. It is recommended that the width, $x_{\text {iris }}$, of the iris be equal to the major radius of the elliptical CSRRs. As can be seen from Fig. 2, rectangular cavities of length $l_{\text {cav }}$, height $h_{\text {cav }}$ and of the same width, $a$, as the feeding waveguide encapsulate the spaces above and below the CSRRs. Without these cavities, a vacuum could not be maintained but the fields present would be similar in character. It is recommended that $l_{\text {cav }}$ minus 2 times the metal thickness, $t$, be only slightly larger than 2 times the minor radius $\left(r_{z}\right)$ of the CSRR. The height of the spaces above and below the CSRRs $\left(h_{\mathrm{cav}}-t\right)$ can be tuned to optimize the deflecting voltage. Table I details the geometry for a designed structure operating at $2.037 \mathrm{GHz}$.

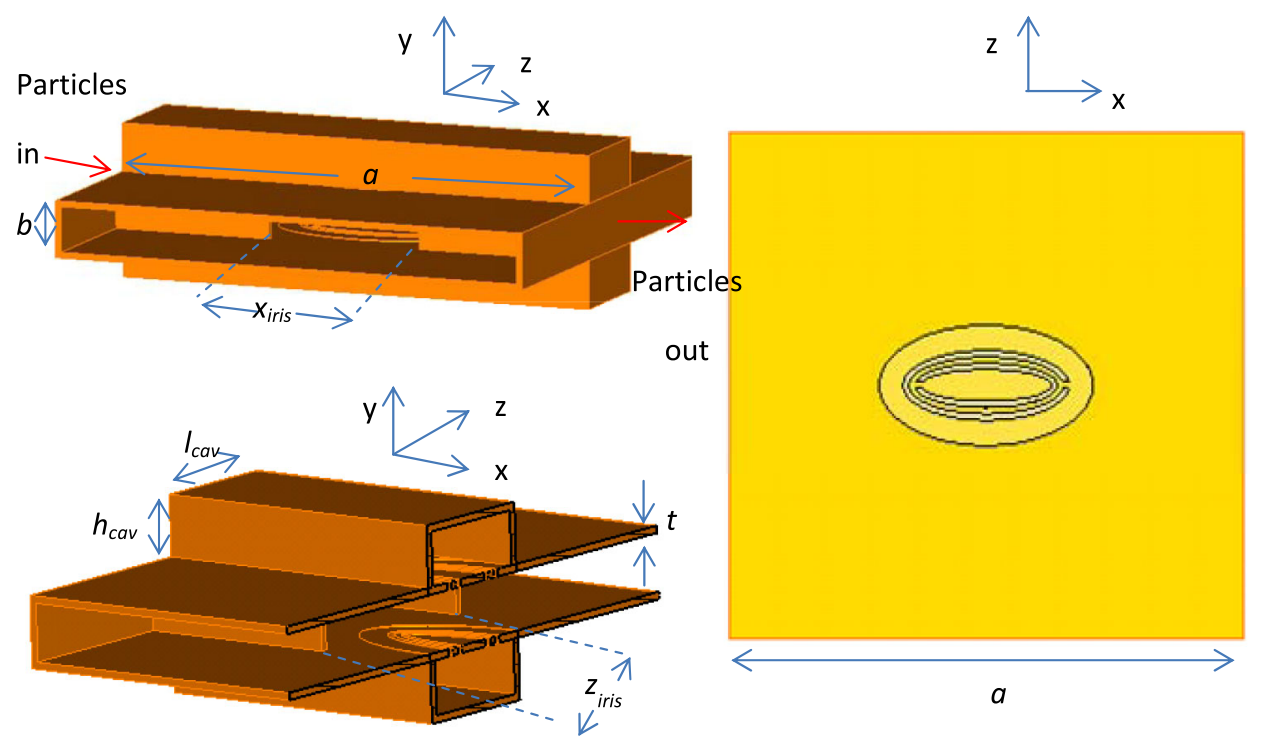

FIG. 2. Perspective (upper left), top view with outer rectangular cavity removed (right) and cut-plane view (bottom left) of the proposed deflecting structure. 


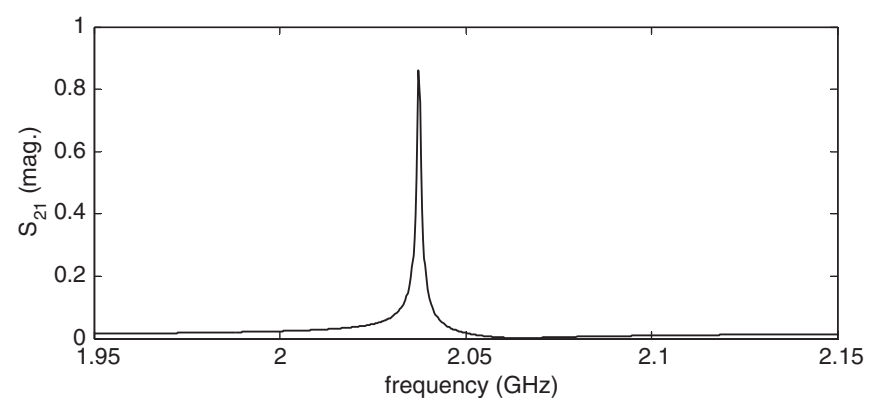

FIG. 3. Power transmission coefficient for the designed deflecting structure.

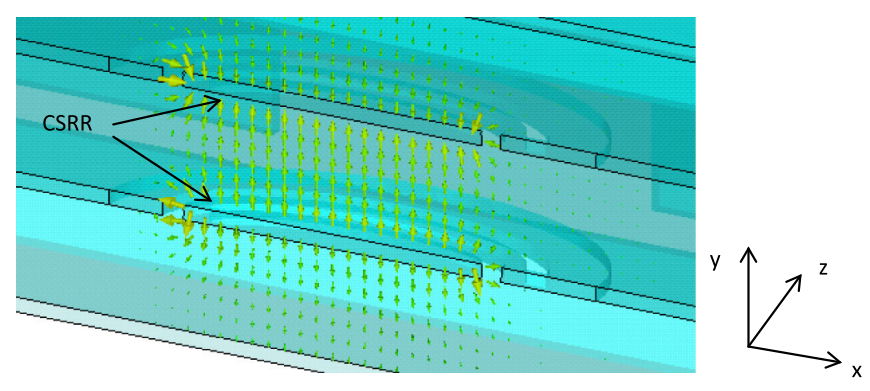

FIG. 4. 2D cut of the electric field at resonance.

\section{EM SIMULATION RESULTS}

A structure defined by the geometry given in Table I has been simulated using CST MICROWAVE STUDIO. The frequency domain, tetrahedral solver was used. An extra fine mesh was applied to the CSRRs and, after some iteration, the mesh adaptation frequency was set close to the operating frequency.

Figure 3 shows the magnitude of the simulated power transmission coefficient for our design. A transmission maximum $\left(S_{21}=0.86\right)$ occurs at $2.037 \mathrm{GHz}$ - the operating frequency.

Figure 4 shows a 2D cut of the electric field at the resonance frequency. The cutting plane is through the midpoint (in the $z$ direction) of the structure. It can be seen that the field is uniform in the $y$ direction. Thus, a particle bunch of some finite radius can be expected to experience the same deflecting field across its entirety. The peak surface field is rather high at $1.172 \sqrt{P_{\text {in }}} \mathrm{MV} / \mathrm{m}-$ where $P_{\text {in }}$ is the power in the excitation signal. From the Kilpatrick criterion [6,7] for copper surfaces, at the operational frequency and assuming a "bravery factor" of 2 , the maximum power which may be safely applied to the structure is, approximately, $4.42 \mathrm{~kW}$. This figure assumes that the maximum tolerable surface E-field is twice (bravery factor $=2$ ) that indicated by the criterion. This increase by a factor of 2 is due to current surface treatment technology not available when the criterion was formulated. The limitation on the maximum value of applied power is the main drawback of the structure. However, the $k$ value mentioned earlier which relates input power to transverse accelerating gradient is high at 23.4. Thus, if the rf power is limited either for hardware or functional reasons, the proposed structure represents a good choice. $R_{\text {shunt }} / Q$ is also high at $821.6 \Omega$ and so the structure is a good choice if the structure should be compact.

Table II presents some key performance metrics for the designed structure. Compared with Refs. [2,3], the total deflecting voltage, at $0.132 \mathrm{MV}$, is considerably less than in [2] (4.9 MV). This is to be expected considering the relatively low rf input power used. In terms of $R_{\text {shunt }} / Q$, we find that our deflector has a higher figure of merit$821.6 \Omega$ compared with $153.8 \Omega$. Compared with [3], the deflecting voltage is comparable- $132 \mathrm{kV}$ compared with $177 \mathrm{kV}$ in [3] - despite operating with around $1 / 20$ th of the rf power. It is also more compact $-85 \mathrm{~mm}$ compared with $350 \mathrm{~mm}$. Again, the $R_{\text {shunt }} / Q$ value is favorable $-821.6 \Omega$ compared with $473.9 \Omega$.

If we compare the deflecting structure with those detailed in [2,3] in terms of beam aperture divided by freespace wavelength we see that this ratio is lowest in our structure: 0.05 compared with 0.36 [2] and 0.43 [3]. This result may explain the higher figures obtained for $R_{\text {shunt }} / Q$ since one would expect the shunt resistance to increase as the size of the beam aperture decreases.

The small beam aperture $(8 \mathrm{~mm})$ is in fact a disadvantage in the sense that particle sources with low emittance must be used and the wakefield effect may be more critical. Another disadvantage is the fact that large losses are incurred in areas of the structure which are inaccessible to cooling mechanisms (i.e. on the CSRRs). However, regarding this latter point, the structure is only compatible with low excitation powers owing to the high surface E-fields and so large amounts of heating will naturally be avoided. In order that any potential heating problems be mitigated it is possible that the structure would have to be operated with low duty cycles.

Another disadvantage is that the configuration of the deflector does not lend itself to multiple-cell operation. This is because the rf power flows transversely to the direction of particle propagation. However, if a total deflecting voltage is required which is greater than one cell

TABLE II. Key performance metrics of deflecting structure.

\begin{tabular}{lccccc}
\hline \hline $\begin{array}{l}\text { Frequency } \\
(\mathrm{GHz})\end{array}$ & $Q$ & $R_{\text {shunt }} / Q(\Omega)$ & $\begin{array}{c}\text { Excitation } \\
\text { power }(\mathrm{kW})\end{array}$ & $\begin{array}{c}\text { Transverse accelerating } \\
\text { gradient }(\mathrm{MV} / \mathrm{m})\end{array}$ & $\begin{array}{c}\text { Deflecting } \\
\text { voltage }(\mathrm{MV})\end{array}$ \\
\hline 2.037 & 1136 & 821.6 & 4.42 & 1.55 & 0.132 \\
\hline \hline
\end{tabular}


can provide, the same configuration as in Fig. 2 can be arrayed in the $x$ direction. Beam pipes or openings in the waveguide walls connecting the various deflecting structures would allow the particles to pass from one cell to the next. The rf power can be distributed via $3 \mathrm{~dB}$ splitters and separately phased so that, as the particle bunch passes from one deflecting structure to the next, it experiences the total deflecting voltage of each cell. A simulation has been performed on two cells coupled together through an aperture in the waveguide wall so the particle bunch can pass from one to the other. It was found that, owing to the presence of the irises, the separate deflecting structures are essentially uncoupled electromagnetically.

\section{ANALYSIS OF RESONANCE FREQUENCY}

We now present an analysis which can predict the operating frequency of the structure. Because of the many free parameters characterizing the CSRR and the need to optimize the deflecting voltage and reduce peak surface fields, achieving a specified operating frequency would require many full wave simulations if no means of prediction were available.

In order to simplify the analysis of the resonance frequency of the structure, we ignore the effect of the inductive irises, the rectangular cavities, and the feeding waveguide, and assume that the final operating frequency is mainly dictated by the geometry of the CSRRs. As is well known [8], the first resonance frequency of the CSRR is characterized by linear voltage and current distributions and can therefore be derived from a quasistatic analysis based on the per-unit-length capacitance and inductance of the structure. These per-unit-length values are then converted to lumped values by multiplying by the effective circumference of the resonator.

We begin by equating the outermost circumference of the elliptical resonator to the circumference of a circular resonator:

$$
2 \pi r_{\mathrm{eq}}=4 r_{x} E\left(\sqrt{1-\frac{r_{z}^{2}}{r_{x}^{2}}}\right),
$$

where $E$ (modulus) is a complete elliptic integral of the second kind. The "equivalent" circular radius, $r_{\text {eq }}$, is then

$$
r_{\mathrm{eq}}=\frac{2}{\pi} r_{x} E\left(\sqrt{1-\frac{r_{z}^{2}}{r_{x}^{2}}}\right) .
$$

At the resonance frequency, current flows from the surrounding metallization through the inductive metal connection on the left (see Fig. 1), splits when it meets the two transmission line branches, and then recombines at the inductive connection on the right and flows into the central patch of metal. Thus, the central metal patch can be at a different potential from the surrounding metallization. The fields on the CSRR therefore differ from that of two

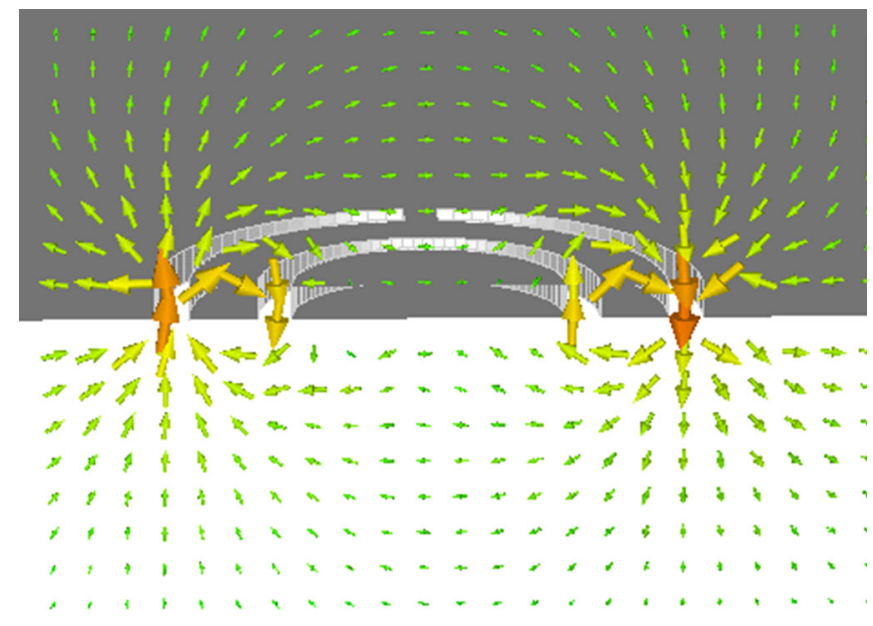

FIG. 5. 2D cut of the CSRR magnetic field at resonance.

semicircular Coplanar waveguide-like transmission lines tracks.

Figure 5 shows a 2D cut of the $\mathrm{H}$-field along the middle of a circular CSRR at resonance. It can be seen that at the CPW-like tracks on the left and right side of the structure, the H-field circulates around the current carrying "signals" of the effective transmission lines but also encircles the central patch of metal. The total inductance can therefore be decomposed into two separate parts. The first is the contribution from the two CPW-like branches. These connect in parallel. The second is the contribution from the $\mathrm{H}$-field encircling the central patch of metal and adds in series. To explain how this series inductance is calculated, we now examine the E-field of the CSRR.

Figure 6 shows that the E-field very closely resembles the so-called "even" mode in a CPW (dominant CPW mode) transmission line but with the central patch of metal acting as the signal and the surrounding waveguide metal as the ground plane. The tracks in between are at a similar potential to the central patch. Therefore, a $2 \mathrm{D}$ cut of the

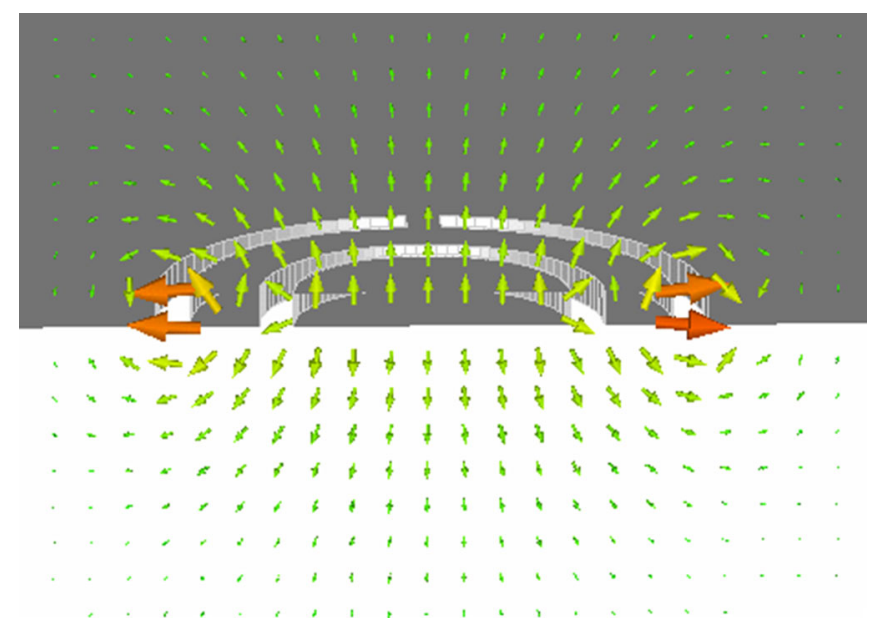

FIG. 6. 2D cut of the CSRR electric field at resonance. 
whole structure (from the point of view of the E-field) looks similar to that of a CPW line with a signal width equal to the radius of the circular patch plus $(s+w)$ or, equivalently, $\left(r_{\mathrm{eq}}-s\right)$. The E-field looks the same if $2 \mathrm{D}$ cuts are taken as shown in Fig. 6 and then rotated around the $y$ axis (the horizontal direction in Fig. 6). This leads us to make an analogy with a disk capacitor.

The capacitance of a parallel plate circular disk capacitor, neglecting fringing fields, is $\varepsilon_{0} \frac{\pi r^{2}}{h}$ [9] or $\varepsilon_{0} \frac{r}{h}(\pi r)$, where $r$ is the radius of the plates and $h$ the distance between them. This latter result is the same as $\pi r$ times half the 2D capacitance of an entire cross section. Therefore, if we find the equivalent parallel plate capacitance of a $2 \mathrm{D}$ cut of the structure, which we will call $C_{\text {pul2 }}$, we can divide it by two and use it to replace the quantity $\varepsilon_{0} r / h$. With $r=\left(r_{\mathrm{eq}}-s\right)$ this gives the capacitance as $\pi\left(r_{\mathrm{eq}}-s\right) C_{\mathrm{pul} 2} / 2$. This corresponds to a straight $\mathrm{CPW}$ line of length $\pi\left(r_{\mathrm{eq}}-s\right) / 2$. This equivalent straight CPW line allows us to calculate the inductance associated with the $\mathrm{H}$-field circulating around the central patch of metal i.e. $\pi\left(r_{\mathrm{eq}}-s\right) L_{\mathrm{pul} 2} / 2$, where $C_{\mathrm{pul} 2}$ and $L_{\text {pul2 }}$ are related by the speed of light.

To summarize, we propose to treat the capacitance and inductance associated with the central patch as if they were created by a straight CPW line with slots of width $s$, a conductor width equal to $\left(r_{\mathrm{eq}}-s\right)$, and with a length $\pi\left(r_{\mathrm{eq}}-s\right) / 2$. This proposed equivalence is highly approximate but, as we will see, leads to accurate results. It also avoids the need to solve the exact 3D mixed boundary problem which would not, in any case, lead to an exact and simple closed form expression.

First, we calculate the inductance of the upper and lower branches of the resonator. The per-unit-length capacitance of a coplanar waveguide plus an additional term for the effective parallel plate capacitors formed by the finite metallization is given by [10]

$$
C_{\text {pul } 1}=4 \varepsilon_{0}\left(\frac{K\left(k_{1}\right)}{K\left(k_{1}{ }^{\prime}\right)}+\frac{1}{2} \frac{t}{s}\right),
$$

where $K(k)$ is a complete elliptic integral of the first kind. Its argument, $k_{1}$, is the elliptic modulus while $k_{1}{ }^{\prime}$ is the complementary elliptic modulus. The addition of the second term in the right-hand side of (3) - for the finite metal thickness - assumes that the fields of the top and bottom faces of the effective transmission line and of the parallel metal faces formed by the metal thickness are independent of each other. Of course, this is not true in reality but the expressions for the exact conformal map of the structure would be so complicated that the analysis would not be worthwhile. The modulus, $k$, and its complement, $k^{\prime}$, are given by (4) and (5), respectively,

$$
k_{1}=\frac{0.5 w}{0.5 w+s}
$$

$$
k_{1}^{\prime}=\sqrt{1-k_{1}^{2}} .
$$

The per-unit-length inductance is given, from basic transmission line theory, as

$$
L_{\mathrm{pul} 1}=\frac{1}{c^{2} C_{\mathrm{pul} 1}}
$$

in which $c$ is the speed of light in a vacuum. (6) is the perunit-length inductance associated with the two semicircular CPW branches on either side of the structure. The radius followed by the signal trace of these branches is

$$
r_{\mathrm{eff}}=r_{\mathrm{eq}}-s-\frac{w}{2},
$$

while the effective angle the transmission line covers (which differs from $2 \pi$ because of the shunt inductive connections of width $g$ ) is given, approximately, by

$$
\theta \approx 2 \pi-2 \frac{g}{r_{\mathrm{eq}}} .
$$

The total inductance contributed by the two semicircular CPW-like branches, which add in parallel, is

$$
L_{1}=\theta r_{\mathrm{eff}} \frac{L_{\text {pul1 }}}{4} .
$$

The per-unit-length capacitance associated with the effective CPW formed by the central patch of metal and the surrounding waveguide metal is

$$
C_{\mathrm{pul} 2}=4 \varepsilon_{0}\left(\frac{K\left(k_{2}\right)}{K\left(k_{2}{ }^{\prime}\right)}+\frac{1}{2} \frac{t}{s}\right)
$$

with the elliptic modulus given by

$$
k_{2}=\frac{r_{\mathrm{eq}}-s}{r_{\mathrm{eq}}} .
$$

The complementary modulus is given by (5) but with $k_{2}$ replacing $k_{1}$. The associated per-unit-length inductance, $L_{\text {pul2 }}$, is given by (6) but with $C_{\text {pul2 }}$ replacing $C_{\text {pul1 } 1}$.

The capacitance and associated inductance of the central patch are given by (12) and (13), respectively,

$$
\begin{gathered}
C=\frac{\theta C_{\mathrm{pul} 2}\left(r_{\mathrm{eq}}-s\right)}{4}, \\
L_{2}=\frac{\theta \pi L_{\mathrm{pul} 2}\left(r_{\mathrm{eq}}-s\right)}{4} .
\end{gathered}
$$

Neglecting the effect of the gap, (12) and (13) reduce to $\pi\left(r_{\mathrm{eq}}-s\right) C_{\mathrm{pul} 2} / 2$ and $\pi\left(r_{\mathrm{eq}}-s\right) L_{\mathrm{pul} 2} / 2$, respectively, as previously discussed.

To calculate the inductance of the narrow tracks, of width $g$, connecting from the surrounding waveguide metal and to the central patch of metal, we use [11] 
TABLE III. Comparison between simulated and calculated CSRR resonance frequencies. All dimensions are in $\mathrm{mm}$.

\begin{tabular}{lllccccc}
\hline \hline$r_{x}$ & $r_{z}$ & $w$ & $s$ & $g$ & $t$ & $\begin{array}{c}f_{0} \text { simulated } \\
(\mathrm{GHz})\end{array}$ & $\begin{array}{c}f_{0} \text { calculated } \\
(\mathrm{GHz})\end{array}$ \\
\hline 4 & 4 & 0.4 & 1.2 & 0.4 & 0.1 & 5.96 & 5.92 \\
6 & 6 & 2.5 & 0.5 & 1 & 1 & 4.08 & 4.11 \\
6 & 6 & 1 & 1 & 1 & 1 & 4 & 4.01 \\
6 & 9 & 1 & 1 & 1 & 1 & 2.93 & 2.98 \\
1 & 1 & 0.1 & 0.05 & 0.1 & 0.01 & 19.1 & 19.4 \\
1 & 5 & 0.1 & 0.05 & 0.1 & 0.01 & 5.19 & 5.08 \\
8 & 8 & 2 & 1 & 1 & 0.5 & 2.94 & 2.88 \\
8 & 14 & 2 & 1 & 1 & 0.5 & 2 & 1.91 \\
6 & 6 & 2 & 0.2 & 1 & 2 & 4.1 & 3.99 \\
6 & 13 & 2 & 0.2 & 1 & 2 & 2.45 & 2.3 \\
\hline \hline
\end{tabular}

$L_{\mathrm{gap}}=2 \times 10^{-7}\left[s \ln \left[\frac{s+\sqrt{s^{2}+\rho_{\mathrm{eq}}^{2}}}{\rho_{\mathrm{eq}}}\right]-\sqrt{s^{2}+\rho_{\mathrm{eq}}^{2}}+\rho_{\mathrm{eq}}\right]$.

This is the inductance of a circular wire of radius $\rho_{\text {eq }}$ and length $s$ at frequencies where the skin effect predominates (negligible internal field in the wire) and so differs from that in [11] by an $s / 4$ term. In order to relate the rectangular cross section of our conductors to that of a circular wire with an equivalent radius $\rho_{\text {eq }}$ we make use of the results in [12] and note that their analytical results are well approximated by the following straight line:

$$
\rho_{\text {eq }}=0.28675(g+t) \text {. }
$$

Provided the ratio $t / g$ is not well below 0.1 , the accuracy of (15) compared with the exact results in [12] is much better than $1 \%$. Use of (15) replaces a long and complicated expression at the expense of some loss in accuracy.

Finally, the total lumped inductance is

$$
L=L_{1}+L_{2}+2 L_{\text {gap }}
$$

and the resonance frequency of the CSRR is

$$
f_{0}=\frac{1}{2 \pi \sqrt{L C}}
$$

Table III shows a comparison between calculated and simulated resonance frequencies for some selected circular and elliptical geometries. The first two entries demonstrate the accuracy achieved for different $w / s$ ratios.
The remaining entries alternate between circular and elliptical shapes for identical $w, s, g$, and $t$ values. It can be seen that the agreement for each entry is very good even for frequencies around $20 \mathrm{GHz}$ but that the great simplification made by the treatment of the elliptical resonators as circular with an equivalent radius given by (2) leads to an increase in the error. The error increases as the major radius becomes larger with respect to the minor but it can be seen that the agreement is still good at $r_{x} / r_{z}=2$ and larger. The greatest error is in the last two entries where the ratio $w / s$ is very large but predicted frequencies are still accurate to within $6.5 \%$ when the major radius is slightly larger than 2 times the minor. All the other entries are accurate to within $2 \%$ for circular geometries and 5\% for elliptical. The formulas also become less accurate when $g$ becomes increasingly large compared with the other dimensions. This is not surprising as (8) is only an approximation involving a change in arclength and assumes a gap which grows radially with increasing distance from the center of the CSRR rather than being completely constant in the $x$ direction (see Fig. 1). For more details on the simulation of the CSRR resonance frequency, see [8].

The calculated value of resonance frequency for the deflecting structure is $2.039 \mathrm{GHz}$. This compares well with the simulated value of $2.037 \mathrm{GHz}$. This result indicates that, at lower frequencies, the effect of the feeding waveguide, the inductive irises, and the outer cavities can be effectively ignored. In Table IV, we compare the calculated and simulated resonance frequency for two different deflecting structures-one operating at $3.13 \mathrm{GHz}$ and the other at $1.58 \mathrm{GHz}$. Again, the calculated frequencies are close to the simulated values. The accuracy of these additional results again suggests that the final operating frequency is mainly determined by the geometry of the CSRRs.

The many approximations made in the preceding analysis result in relatively simple expressions which nevertheless lead to good accuracy. As previously discussed, the formulas are useful when designing the structure for a specified operating frequency as they illustrate the relationship between the CSRR's geometry and the operating frequency. This allows a good initial guess of the geometry required to satisfy a specified operating frequency. It also cuts the number of potentially time consuming full wave simulations required to tune the structure. This is especially true if the deflecting voltage and peak fields are to be optimized. Generally speaking, the peak surface field strength increases with decreasing $s$ and the deflecting

TABLE IV. Comparison between simulated and calculated deflecting structure operating frequencies.

\begin{tabular}{llllllllllllll}
\hline \hline$r_{x}$ & $r_{z}$ & $w$ & $s$ & $g$ & $t$ & $a$ & $b$ & $l_{\text {cav }}$ & $h_{\text {cav }}$ & $x_{\text {iris }}$ & $z_{\text {iris }}$ & Simulated $f_{0}(\mathrm{GHz})$ & Calculated $f_{0}(\mathrm{GHz})$ \\
\hline 14 & 7 & 1.5 & 1.5 & 1 & 1 & 85 & 8 & 20 & 9.5 & 14 & 22 & 2.037 & 2.039 \\
8.5 & 4.5 & 0.4 & 0.4 & 0.5 & 1 & 60 & 8 & 12 & 13 & 8.5 & 8.5 & 3.13 & 3.15 \\
20 & 6 & 1.5 & 1.5 & 1 & 1 & 120 & 8 & 28 & 13 & 16 & 16 & 1.58 & 1.49 \\
\hline \hline
\end{tabular}


voltage increases as the major radius of the resonators is made larger and the minor radius smaller.

\section{REDUCING THE PEAK SURFACE ELECTRIC FIELD}

The highest surface E-field occurs at the CSRR. It can be reduced by blending the corners of the CSRR metallization to achieve a more rounded cross section. Figure 7 shows the effect of blending the edges of the CSRR defined in Table I with a "blend radius" of $0.5 \mathrm{~mm}$. The resulting circular cross section at the corners has the same shape as one quadrant of a circle of radius $0.5 \mathrm{~mm}$.

To illustrate the effect of blend radius on performance we summarize, in Table $\mathrm{V}$, the performance characteristics of the structure defined in Table I as the metal tracks change from a rectangular shape to having a blend radius $\left(r_{\text {blend }}\right)$ of $0.25 \mathrm{~mm}$ and then $0.5 \mathrm{~mm}$.

It can be seen that the maximum rf power, $P_{\text {in }}$, that can be safely applied (assuming twice the fields indicated by Kilpatrick's criterion) increases by a factor of around 6.6. Also, the parameter $R_{\mathrm{sh}} / Q$ increases by around $20 \%$. The results also suggest that the total deflecting voltage $\left(V_{\text {def }}\right)$ and transverse accelerating gradient $\left(G_{t}\right)$ can be increased by a factor of approximately 2.7. The difference between a blend radius of $1 / 4(0.25 \mathrm{~mm})$ and $1 / 2(0.5 \mathrm{~mm})$ of the conductor thickness is seen to be small in terms of the maximum safe rf power, deflecting voltage and gradient but still leads to a significant increase in $R_{\mathrm{sh}} / Q$. The results are explained by the fact that the deflecting voltage (for a fixed rf power) and the $Q$ factor increase only by a very small amount as the blend radius increases but the dissipated power reduces considerably. There is a large reduction in peak surface fields when the blend radius changes from 0 to $0.25 \mathrm{~mm}$ and a small reduction when it changes from 0.25 to $0.5 \mathrm{~mm}$. Rounding off the edges of the metal has an effect on the resonance frequency. With a blend radius of $0.5 \mathrm{~mm}$, the operating frequency is slightly reduced-from 2.04 to $1.99 \mathrm{GHz}$ - but the formulas presented in the previous section can still be used as a good approximation.

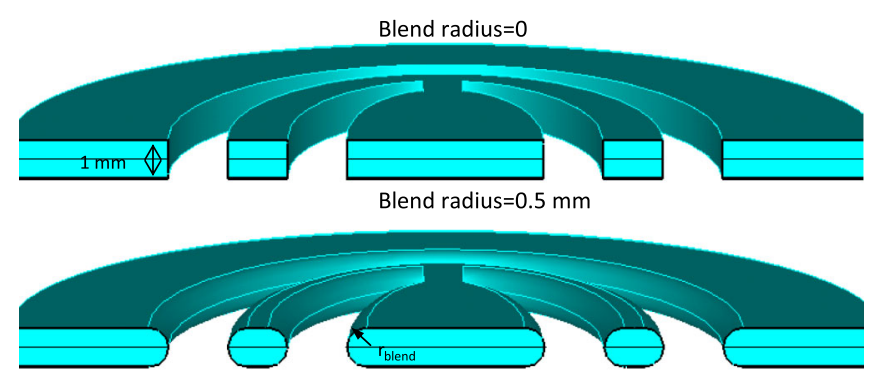

FIG. 7. Cross section of the CSRR used in the deflecting cavity with rectangular conductors (top) and edges smoothed with a blend radius of $0.5 \mathrm{~mm}$ (bottom).
TABLE V. Effect of rounding the CSRR metallization edges on deflector performance characteristics. The other deflector geometrical parameters are: $r_{x}=14, r_{z}=7, w=1.5, s=$ $1.5, g=1, t=1, a=85, b=8, l_{\text {cav }}=20, h_{\text {cav }}=9.5, x_{\text {iris }}=$ $14, z_{\text {iris }}=22$. All dimensions are in $\mathrm{mm}$.

\begin{tabular}{lccccc}
\hline \hline$r_{\text {blend }}$ & $R_{\text {sh }} / Q(\Omega)$ & $P_{\text {in }}(\mathrm{kW})$ & $V_{\text {def }}(\mathrm{MV})$ & $G_{t}(\mathrm{MV} / \mathrm{m})$ & $\begin{array}{c}\text { Simulated } \\
f_{0}(\mathrm{GHz})\end{array}$ \\
\hline 0 & 821.6 & 4.41 & 0.132 & 1.55 & 2.037 \\
0.25 & 867.7 & 29.2 & 0.346 & 4.07 & 2.01 \\
0.5 & 982 & 29.75 & 0.356 & 4.19 & 1.99 \\
\hline \hline
\end{tabular}

\section{CONCLUSIONS}

We have presented simulation results for a normalconducting rf deflecting structure which utilizes the magnified electric fields of a pair of CSRRs. The structure is simple and compact ( $85 \mathrm{~mm}$ along particle path). The beam aperture is restricted to $8 \mathrm{~mm}$ but the main drawback is that the high fields limit the input power to $4.42 \mathrm{~kW}$. However, the deflecting voltage $(0.132 \mathrm{MV})$ and transverse accelerating gradient $(1.55 \mathrm{MV} / \mathrm{m})$ are relatively high. These features may make the deflector attractive for low energy accelerator applications in which the structure's size and cost need to be minimized. We have also presented, as a design aid, relatively uncomplicated yet accurate expressions which allow the operating frequency to be predicted. They can therefore reduce the number of full wave simulations required to design and optimize the deflector. Furthermore, by rounding off the edges of the CSRR metallization, we have shown that the peak surface fields can be reduced, the maximum safe rf power increased, and the resulting deflecting voltage and gradient increased to $0.356 \mathrm{MV}$ and $4.19 \mathrm{MV} / \mathrm{m}$ respectively.

\section{ACKNOWLEDGMENTS}

We thank the Science and Technology Facilities Council, UK, for financial support.

[1] C. H. Schaefer, J. Vac. Sci. Technol. B 4, 1236 (1986).

[2] D. La Civita, P. Craievich, Y. A. Kharoubi, and G. Penco, in Proceedings of the International Particle Accelerator Conference, Kyoto, Japan (ICR, Kyoto, 2010), MOPD097.

[3] J. P. Dai, J. R. Zhang, G. X. Pei, M. Hou, Q. Gu, M. H. Zhao, and S.P. Zhong, in Proceedings of the 31st International Free Electron Laser Conference (FEL 09), Liverpool, UK (STFC Daresbury Laboratory, Warrington, 2009), TUPC24.

[4] F. Falcone, T. Lopetegi, J. D. Baena, R. Marques, F. Martin, and M. Sorolla, IEEE Microw. Wirel. Compon. Lett. 14, 280 (2004).

[5] J. B. Pendry, A. J. Holden, D. J. Robbins, and W. J. Stewart, IEEE Trans. Microwave Theory Tech. 47, 2075 (1999).

[6] W. D. Kilpatrick, Rev. Sci. Instrum. 28, 824 (1957).

[7] T. J. Boyd, Jr., Los Alamos Group Report No. AT-1:82-28, 1982. 
[8] J. D. Baena, J. Bonache, F. Martin, R. Marqués, F. Falcone, T. Lopetegi, M. A. G. Laso, J. García, I. Gil, and M. Sorolla, IEEE Trans. Microwave Theory Tech. 53, 1451 (2005).

[9] I. Wolff and N. Knoppik, IEEE Trans. Microw. Theory Techniques, 22, 857 (1974) [http://ieeexplore.ieee.org/xpl/ articleDetails.jsp?arnumber=1128364].

[10] C. P. Wen, IEEE Trans. Microwave Theory Tech. 17, 1087 (1969).
[11] E. B. Rosa, in The Self and Mutual Inductances of Linear Conductors, Natl. Bur. Stand. (U.S.) Circular No. 4 (U.S. GPO, Washington, DC, 1908), pp. 301-344, Scientific Paper 80 (S80).

[12] K. F. Goddard, A. A. Roy, and J. K. Sykulski, Measurement Technol. 152, 7 (2005) [http://ieeexplore.ieee.org/xpl/ articleDetails.jsp?tp $=\&$ arnumber $=1394001 \&$ queryText $\% 3$ Dinductance+and+resistance+calculations+for+isolated]. 